\title{
Cavernous Sinus Thrombosis as Complications of Cellulitis Orbital: Case Report
}

\author{
Astari Arum Cendani Goller ${ }^{1}$, Kumara Tini² \\ ${ }^{1,2}$ Department of Neurology, Faculty of Medicine, Udayana University / Sanglah General Hospital Denpasar \\ Corresponding Author: Astari Arum Cendani Goller
}

\begin{abstract}
Background: Cavernous sinus thrombosis is a complication of infection around the face or paranasal sinuses with high morbidity and mortality rates. Cavernous sinus thrombosis is generally caused by septic infection, such as orbital cellulitis.

Case Illustrations: A 35-year-old male who came to the neurology polyclinic complained of swelling in the left eyelid, red, watery eyes, blurred vision, difficulty opening the left eye, and the movement of the left eyeball felt normal. The patient had a history of left orbital cellulitis. Laboratory investigations showed an increase in inflammatory markers. The CT scan with contrast showed a hyperdense lesion in the left orbit, suggesting orbital cellulitis. The patient was diagnosed with a cavernous sinus thrombosis due to orbital cellulitis. He was treated with an antibiotic, anticoagulant, and corticosteroid. Three months after treatment, the visual complaints improved, the headache decreased drastically, and we did not find any extraocular muscles weakness.

Conclusions: The administration of antibiotics and anticoagulant therapy in patients with cavernous sinus thrombosis has been proven clinically. The use of corticosteroids has not been proven but is strongly suspected to reduce edema caused by compression of the cavernous sinus.
\end{abstract}

Keywords: Cavernous sinus thrombosis, orbital cellulitis, infections

\section{BACKGROUND}

Cavernous sinus thrombosis is a rare, life-threatening condition, as the complication of head infection or after traumatic injury or surgery, especially in patients with thrombophilic disorders.[1] The cause of cavernous sinus thrombosis is usually septic but can also be aseptic. Most infections are caused by Staphylococcus aureus (60-70\%); fungal infections are only found in immunocompromised individuals. $[2,3]$

The early symptoms of cavernous sinus thrombosis are fever, headache, periorbital swelling, and ophthalmoplegia. Although it can be treated with antibiotics and anticoagulation, the risk of long-term sequelae, such as vision, diplopia, and stroke, remains significant. There are residual symptoms in $50 \%$ of patients, such as third or sixth nerve palsy.[1]

Complications include sepsis, meningitis, subdural empyema, brain abscess, blindness, panhypopituitarism, intracranial hypertension, infectious arteritis, internal carotid artery mycotic aneurysm, and vasospasm, septic embolism, stroke due to carotid constriction, cortical vein thrombosis, or hemorrhage, and death. $[1,2]$

We report a patient with cavernous sinus thrombosis as a complication of orbital cellulitis.

\section{CASE ILLUSTRATION}

A 35-year-old male came to the neurology polyclinic with a history of headaches since 11 months earlier, throbbing, especially on the left side, and sometimes radiating to the left eye, with mild intensity. The pain improves with 

report.

analgetic. This complaint is also accompanied by the blurred and double vision in the left eye. Patients with a history of left orbital cellulitis complained of swelling in the left eyelid, red, watery eyes, blurred vision, difficulty opening the left eye, and the movement of the left eyeball felt normal. The patient also complained of a history of fever. (Figure 1.)

Vital signs within normal limits. Examination revealed decreased visual acuity in the left eye of $1 / 60$, ptosis of the left eye, paresis of the oculomotor nerve, abducens nerve, and left trochlear nerve (Fig. 2.). Fundoscopic examination revealed papilledema in the left eye. On laboratory examination, we found an increase in inflammatory markers such as leukocytes 27000, ESR 52.2mm/hour, CRP $83.83 \mathrm{mg} / \mathrm{dl}$, D-dimer 0.67. The CT scan without contrast showed a hyperdense lesion in the left orbit, suggesting orbital cellulitis. The DSA cerebral examination showed left cavernous sinus thrombosis with venous rerouting to the vein of Labbe with the antegrade flow without cortical venous reflux. (Figure 3.)

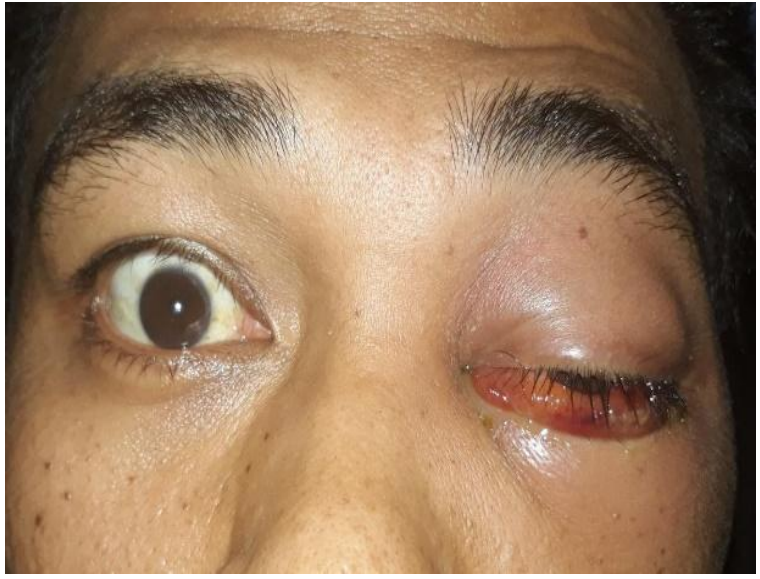

Figure 1. Patients with complaints of swelling in the left eyelid, red, watery eyes.

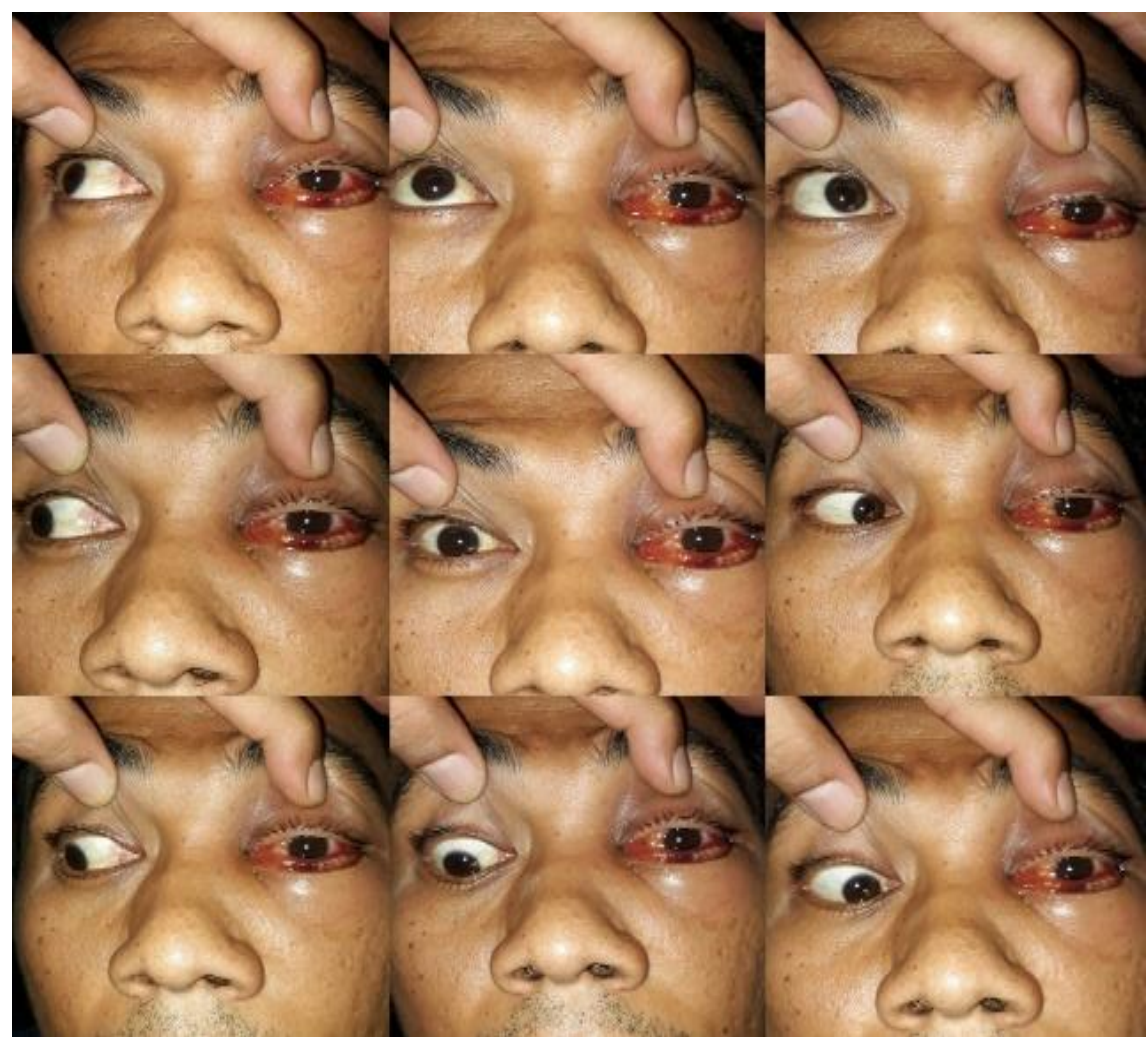

Figure 2. On examinations showed paresis of the oculomotor nerve, abducens nerve, and left trochlear nerve

The patient was diagnosed with a cavernous sinus thrombosis due to orbital cellulitis. He was treated with antibiotics ceftriaxone 2 grams every 12 hours, anti- platelet aggregation Acetosal $80 \mathrm{mg} / 24$ hours, and corticosteroids $62.5 \mathrm{mg} / 12$ hours for two weeks. 

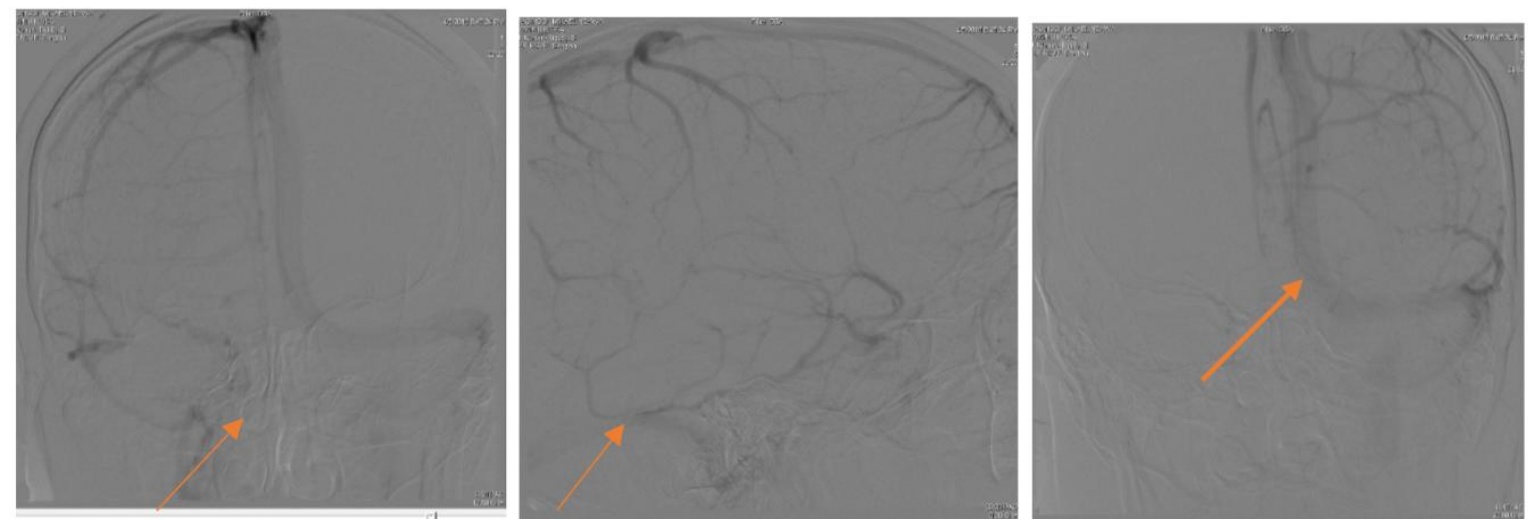

Figure 3. The DSA cerebral examination showed left cavernous sinus thrombosis with venous rerouting to the vein of Labbe with the antegrade flow without cortical venous reflux.

Three months after treatment, the patient came for following up. On examination, the visual complaints improved, the headache decreased drastically, and we did not find any extraocular muscles weakness. We continued anticoagulant therapy and planned another MRI examination.

\section{DISCUSSION}

Cavernous sinus thrombosis is a complication of infection around the face or paranasal sinuses with a high morbidity and mortality rate.[4] The rare incidence and overlapping complaints of eye disease are generally at risk of delaying diagnosis and resulting in increased morbidity.[5]

Cavernous sinus thrombosis is generally caused by septic infection, including infections around the face, especially within the "triangle of the face," including abscesses or cellulitis, dental sinusitis, otitis media, and mastoiditis. Aseptic causes are less common such as trauma, surgery, or pregnancy.[3,6]

The patient presented with fever, headache, periorbital edema, and subacute ophthalmoplegia. The most common neurologic symptoms (90\%) were periorbital edema, chemosis, ptosis, proptosis, and ophthalmoplegia. Symptoms rarely reported, such as papilledema, retinal hemorrhages, decreased vision, photophobia, and slow pupillary reflexes. Most of the patients complained of headaches and cranial nerve paresis $(50 \%$ to $80 \%$ ). In general, paresis of nerve VI is the most common paresis, causing partial ophthalmoplegic disorders. However, it can progress to external ophthalmoplegia with paresis of nerves III, IV, and VI, and sensory of nerve V. Horner's syndrome is sometimes present.[6,7]

A non-contrast CT scan of the head may show several abnormalities such as vascular edema, protrusion of the lateral margin of the cavernous sinus, exophthalmos, and the presence of sphenoid or ethmoid sinusitis, or mass lesions near the sphenoid or pituitary gland.[4] There were signs of inflammation in an increase in leukocytes, CRP, ESR, and D-dimer on laboratory examination. On examination of pus culture, bacterial growth was found. A lumbar puncture examination is important to rule out the possibility of cerebral infection such as meningitis.[3]

Antibiotics and anticoagulants are primary therapy. Antibiotic therapy is recommended for 3 to 4 weeks. The use of an anticoagulant, such as UFH or LMWH, is recommended for several weeks to several months.[7] A retrospective clinical trial showed a reduction in mortality from $40 \%$ to $14 \%$ with UFH and neurologic morbidity from $61 \%$ to $31 \%$ when combined with antibiotics. Administration of corticosteroids reduces inflammation and vasogenic edema around cranial nerves and orbital structures. Surgical intervention is not recommended for the cavernous sinus. However, some special conditions require sphenoidectomy, ethmoidectomy, maxillary antrostomy, mastoidectomy, abscess 

report.

drainage, craniotomy (subdural empyema), orbital decompression, or placement of a VP shunt. [6,7]

The differential diagnosis of cavernous sinus syndrome can also be due to local compression of the cavernous sinus from non-infectious and non-thrombotic causes, $30 \%$ of which are tumors. Causes of pain in ophthalmoplegia include orbital cellulitis, orbital apex syndrome, tuberculosis, sarcoidosis, syphilis. [6,7]

Acknowledgement: None

\section{Conflict of Interest: None}

\section{Source of Funding: None}

\section{REFERENCES}

1. Caso V,Agnelli G, Paciaroni M. Handbook on Cerebral Venous Thrombosis.Font Neurol neurosci,Karger.2008;23:1-111

2. Bousser,MG,Ferro,JM.2007.cerebral venous thrombosis:an update.Lancet Neurologi. 2007; 6:162-170
3. Plewa MC, Gupta M. Cavernous Sinus Thrombosis. [Updated 2019 Feb 23]. In: StatPearls

4. Matthew TJH, Hussein A. Atypical Cavernous Sinus Thrombosis: A Diagnosis Challenge and Dilemma. Cureus. 2018 Dec 04;10(12):e3685

5. Dolapsakis C, Kranidioti E, Katsila S, Samarkos M. Cavernous sinus thrombosis due to ipsilateral sphenoid sinusitis. BMJ Case Rep. 2019 Jan 29;12(1)

6. Hen MC, Ho YH, Chong PN, Chen JH. A rare case of septic cavernous sinus thrombosis as a complication of sphenoid sinusitis. Ci Ji Yi Xue Za Zhi. 2019 JanMar;31(1):63-65.

7. Frank GS, Smith JM, Davies BW, Mirsky DM, Hink EM, Durairaj VD. Ophthalmic manifestations and outcomes after cavernous sinus thrombosis in children. $\mathrm{J}$ AAPOS. 2015 Aug;19(4):358-62

How to cite this article: Goller AAC, Tini K. Cavernous sinus thrombosis as complications of cellulitis orbital: case report. International Journal of Research and Review. 2021; 8(10): 248-251. DOI: https://doi.org/10.52403/ijrr. 20211032 